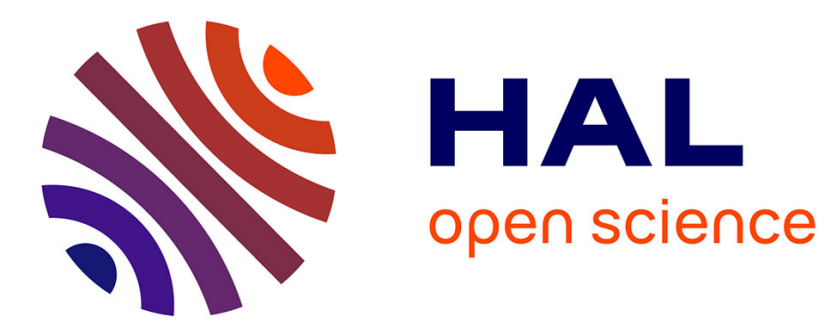

\title{
Towards holographic spintronics
}

Koji Hashimoto, Norihiro Iizuka, Taro Kimura

\section{To cite this version:}

Koji Hashimoto, Norihiro Iizuka, Taro Kimura. Towards holographic spintronics. Physical Review D, 2015, 91, pp.086003. 10.1103/PhysRevD.91.086003 . hal-01140007

\section{HAL Id: hal-01140007 https://hal.science/hal-01140007}

Submitted on 7 Apr 2015

HAL is a multi-disciplinary open access archive for the deposit and dissemination of scientific research documents, whether they are published or not. The documents may come from teaching and research institutions in France or abroad, or from public or private research centers.
L'archive ouverte pluridisciplinaire HAL, est destinée au dépôt et à la diffusion de documents scientifiques de niveau recherche, publiés ou non, émanant des établissements d'enseignement et de recherche français ou étrangers, des laboratoires publics ou privés. 


\title{
Towards holographic spintronics
}

\author{
Koji Hashimoto, ${ }^{1,2, *}$ Norihiro Iizuka, ${ }^{1,3, \dagger}$ and Taro Kimura ${ }^{4,2, *}$ \\ ${ }^{1}$ Department of Physics, Osaka University, Toyonaka, Osaka 560-0043, JAPAN \\ ${ }^{2}$ Mathematical Physics Laboratory, RIKEN Nishina Center, Saitama 351-0198, JAPAN \\ ${ }^{3}$ Yukawa Institute for Theoretical Physics, Kyoto University, Kyoto 606-8502, JAPAN \\ ${ }^{4}$ Institute de Physique Théorique, CEA Saclay, 91191 Gif-sur-Yvette, FRANCE \\ (Received 5 May 2013; published 6 April 2015)
}

\begin{abstract}
We study transport phenomena of total angular momentum in holography, as a first step toward holographic understanding of spin transport phenomena. Spin current, which has both the local Lorentz index for spins and the space-time vector index for current, couples naturally to the bulk spin connection. Therefore, the bulk spin connection becomes the source for the boundary spin current. This allows us to evaluate the spin current holographically, with a relation to the stress tensor and metric fluctuations in the bulk. We examine the spin transport coefficients and the thermal spin Hall conductivity in a simple holographic setup.
\end{abstract}

DOI: 10.1103/PhysRevD.91.086003

PACS numbers: 11.25.Tq, 85.75.-d, 75.76.+j

\section{INTRODUCTION}

Spintronics is a technology where we manipulate the intrinsic electron spin degrees of freedom instead of the electric charge [1,2]. In ferromagnetic/antiferromagnetic materials, spin-charge separation can occur, and in such a situation, it is useful to consider spin as an independent degree of freedom which carries information. Because electric charge transport is not involved there, spin devices can reduce power consumption compared to usual electric ones and exceed the velocity limit of the electron charge. This spintronics is actually used widely, for example, for read heads of hard drives, and is based on a recent development of experimental technologies manipulating imbalance between up spins and down spins. For these reasons, spin transport phenomena have been attracting special interest recently.

Recent research on the spin transport basically relies on one-body quantum mechanical analyses, especially in the presence of a spin-orbit interaction. However, in strongly correlated systems, we have to go beyond the one-body physics by treating the interaction effect seriously. In this paper, we propose a method to study the spin transport phenomena for strongly correlated systems by using the holography, i.e., gauge/gravity correspondence [3-5]. The method of holography is one of the most useful tools to study strongly correlated quantum field theories. While there are some attempts to include effects of spins in holography, e.g., Refs. [6-14], study of spin transport itself has not yet been performed in the literature. To discuss the spin degrees of freedom, we first show a definition of spin current from a relativistic field theoretical viewpoint as a

\footnotetext{
*koji@phys.sci.osaka-u.ac.jp

iizuka@yukawa.kyoto-u.ac.jp

taro.kimura@riken.jp
}

conserved Nöther's current. Then with this definition, we show how to deal with the spin transport coefficients from the holographic viewpoint. The key point is that the spin connection is naturally regarded as a source for the spin current. We demonstrate a holographic treatment of the spin transport, on a "boosted" Schwarzschild black brane background in anti-de Sitter (AdS), and we calculate a spin transport coefficient and a thermal spin Hall conductivity.

\section{SPIN CURRENT}

The spin current is, as the name suggests, a flow of the intrinsic spin degrees of freedom, instead of the electric charge. If $z$-spin is conserved, namely a good quantum number, we can apply a naive definition of the spin current,

$$
\vec{J}_{z}=\frac{1}{2}\left(\vec{J}_{\uparrow}-\vec{J}_{\downarrow}\right) .
$$

This means that the spin current is given by the difference between flows of up and down spins, $\vec{J}_{\uparrow}$ and $\vec{J}_{\downarrow}$, while the electric current is the total contribution of them, $\vec{J}=\vec{J}_{\uparrow}+\vec{J}_{\downarrow}$, as shown in Fig. 1. This definition (1)
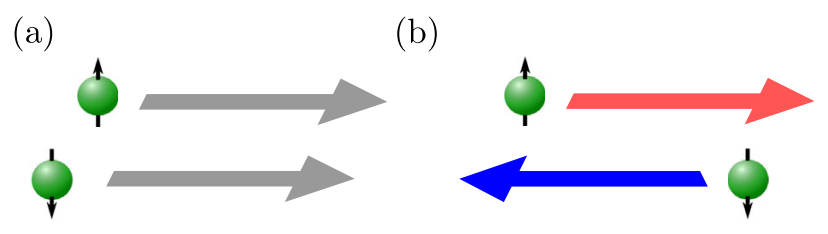

FIG. 1 (color online). (a) The charge current is just the total contribution of up- and down-spin currents $\vec{J}=\vec{J}_{\uparrow}+\vec{J}_{\downarrow}$. (b) The spin current is given by difference between them, $\vec{J}_{z}=\frac{1}{2}\left(\vec{J}_{\uparrow}-\vec{J}_{\downarrow}\right)$. This picture is available if and only if $z$-direction spin is conserved. 
corresponds to the Schwinger representation of the spin operator, $\vec{s}=\frac{1}{2} \psi^{\dagger} \vec{\sigma} \psi$.

The expression (1) is available if and only if the spin is conserved or, at least, approximately conserved [15]. However, generically the electron spin is not conserved by itself, due to the spin-orbit interaction. Therefore, the naive definition of the spin current (1) has to be modified in the presence of such an effect.

First we consider how to define the spin current from the field theoretical point of view. Let us recall the treatment of conserved currents in the context of quantum field theories. A conserved current is defined as a variation of an action with respect to the corresponding source. For example, the electric current $J^{\mu}$ is derived by differentiating an action with respect to a $\mathrm{U}(1)$ gauge field,

$$
J^{\mu}=\frac{\delta S}{\delta A_{\mu}} .
$$

Conservation of $J^{\mu}$ is guaranteed by Nöther's theorem, associated with a U(1) gauge symmetry,

$$
\partial_{\mu} J^{\mu}=0 .
$$

In the weak coupling limit of a $\mathrm{U}(1)$ gauge theory, the $\mathrm{U}(1)$ local symmetry reduces to a global one. The $A_{\mu}$ becomes a nondynamical background gauge potential, which is a source, and the $J^{\mu}$ becomes a global current. In this limit, the global U(1) current $J^{\mu}$ couples to the source $A_{\mu}$ in the Lagrangian as $\mathcal{L}_{\text {source }}=A_{\mu} J^{\mu}$. Therefore, the U(1) current $J^{\mu}$ is obtained by differentiating the action with respect to its source $A_{\mu}$.

Similarly a stress tensor is given by a variation of an action with respect to a metric,

$$
T^{\mu \nu}=\frac{1}{\sqrt{-g}} \frac{\delta S}{\delta g_{\mu \nu}} .
$$

The conservation of energy and momentum

$$
\partial_{\mu} T^{\mu \nu}=0
$$

comes from the translation invariance in temporal and spatial directions, respectively. In the weak gravity limit (where gravity is decoupled), nondynamical background metric $g_{\mu \nu}$ becomes a source for the stress tensor, and it couples with the stress tensor as $\mathcal{L}_{\text {source }}=g_{\mu \nu} T^{\mu \nu}$ in the Lagrangian.

In this way, in order to obtain a conserved quantity, we have to introduce a corresponding field (or source) which couples to the conserved quantity. For the case of the spin current $J^{\mu} \hat{a} \hat{b}$, our claim is that the spin connection $\omega_{\mu}{ }^{\hat{a}} \hat{b}$ is the corresponding field (source). This implies that they couple as $\mathcal{L}_{\text {source }}=\omega_{\mu}{ }^{\hat{a} \hat{b}}{ }^{\mu}{ }_{\hat{a} \hat{b}}$ in the Lagrangian. By differentiating an action with respect to the spin connection, we can obtain the spin current.
To see why it is so, let us recall the nature of spin. The spin operator $s_{\hat{a}}=\sigma_{\hat{a}} / 2$ has an index $\hat{a}$ for the orientation of the spin. Here the hatted index $\hat{a}$ takes only a spatial coordinate as $\hat{a}=\hat{x}, \hat{y}, \hat{z}$, and $\sigma$ is the Pauli matrix. Spin is conserved only in the sense that the total angular momentum is conserved. The total angular momentum is associated with the global rotational symmetry of the system. If we uplift this global rotational symmetry to a local one, then these become a subgroup of the local Lorentz symmetry. Therefore, it is natural to associate the conserved spin $\sigma_{\hat{a}}$ to a local Lorentz generator $\Sigma_{\hat{a} \hat{b}}=\frac{i}{4}\left[\gamma_{\hat{a}}, \gamma_{\hat{b}}\right]$ as $\sigma^{\hat{a}}=\epsilon^{\hat{a} \hat{b} \hat{c}} \sum_{\hat{b} \hat{c}}$, where $\epsilon^{\hat{a} \hat{b} \hat{c}}$ is an antisymmetric tensor taking \pm 1 defined on the spatial part of the local Lorentz indices; i.e., $\hat{a}, \hat{b}, \hat{c}$ of $\epsilon^{\hat{a} \hat{b} \hat{c}}$ takes only $\hat{x}, \hat{y}, \hat{z}$. Furthermore, since the spin connection $\omega_{\mu}^{\hat{a}} \hat{b}$ is a gauge field associated with the local Lorentz symmetry, it is natural to associate it to the conserved spin current $J^{\mu} \hat{a} \hat{b}$, as Eq. (2).

Therefore, we reach a conclusion that a spin current is given by a variation of an action with respect to a spin connection as

$$
J_{\hat{a} \hat{b}}^{\mu}=\frac{\delta S}{\delta \omega_{\mu}^{\hat{a} \hat{b}}} .
$$

From now on, the hatted indices $\hat{a}, \hat{b}, \ldots$ represent the local Lorentz indices, so they stand for $\hat{t}, \hat{x}, \hat{y}, \hat{z}$. Greek indices $\mu, \nu, \ldots$ stand for curved spacetime vector indices. The spin connection is written in terms of a vielbein $e_{\mu}{ }^{\hat{a}}$ as

$$
\begin{aligned}
\omega_{\mu}{ }^{\hat{a} \hat{b}} & =e_{\nu}{ }^{\hat{}} \nabla_{\mu} e^{\nu \hat{b}}=e_{\nu}{ }^{\hat{a}} \partial_{\mu} e^{\nu \hat{b}}+e_{\lambda}{ }^{\hat{a}} \Gamma_{\mu \nu}^{\lambda} e^{\nu \hat{b}} \\
& =-e_{\nu}{ }^{\hat{b}} \nabla_{\mu} e^{\nu \hat{a}}=-\omega_{\mu}{ }^{\hat{b} \hat{a}},
\end{aligned}
$$

where $\Gamma_{\mu \nu}^{\lambda}$ stands for the Christoffel symbol, and the vielbein $e_{\mu}^{\hat{a}}$ satisfies $g_{\mu \nu}=\eta_{\hat{a} \hat{b}} e_{\mu}{ }^{\hat{a}} e_{\nu}^{\hat{b}}$, with the local Lorentz metric $\eta_{\hat{a} \hat{b}}=\operatorname{diag}(-1,1,1,1)$.

Usually, we call the following current as a spin current,

$$
J^{\mu \hat{a}}=\epsilon^{\hat{0} \hat{a} \hat{b} \hat{c}} J^{\mu} \hat{b},
$$

rather than the former one $J^{\mu} \hat{a} \hat{b}$. Here we use the convention $\epsilon^{\hat{0} \hat{1} \hat{2} \hat{3}}=1$. One can easily see that the definition (8) is consistent with, for example, the standard free fermion spin current. To see this, let us consider the generic form of a fermionic Lagrangian on a curved space, which is given by

$$
\mathcal{L}_{\mathrm{F}}=\bar{\psi}\left[i e^{\mu} \hat{a} \gamma^{\hat{a}}\left(\partial_{\mu}-i A_{\mu}-\frac{i}{2} \omega_{\mu}^{\hat{a} \hat{b}} \Sigma_{\hat{a} \hat{b}}\right)-m\right] \psi .
$$

From this, we have the spin current by differentiating it with the spin connection, 


$$
J_{\hat{a} \hat{b}}^{\mu}=\frac{1}{2} \bar{\psi} \gamma^{\mu} \Sigma_{\hat{a} \hat{b}} \psi \longrightarrow J_{\hat{a}}^{\mu}=\frac{1}{2} \bar{\psi} \gamma^{\mu}\left(\sigma_{\hat{a}} \otimes \mathbb{1}\right) \psi .
$$

This is regarded as a current carrying $\hat{a}$-direction spin. We can see that the zeroth component correctly gives the spin density

$$
J_{\hat{a}}^{0}=\psi^{\dagger}\left(s_{\hat{a}} \otimes \mathbb{1}\right) \psi .
$$

In this way, we have seen that the definition (8) is consistent with the conventional one for the spin current. However, it is more convenient to consider $J_{\hat{a} \hat{b}}^{\mu}$ as a spin current, since $J^{\mu}{ }_{\hat{a}}$ defined in Eq. (8) is not local Lorentz invariant tensor. This is because the $\epsilon^{\hat{0} \hat{a} \hat{b} \hat{c}}$ tensor takes explicit index component $\hat{0}$.

The conservation of the spin current $J^{\mu} \hat{a} \hat{b}$,

$$
\partial_{\mu} J_{\hat{a} \hat{b}}^{\mu}=0,
$$

is associated with the local Lorentz invariance, and the spin current $J_{\hat{a} \hat{b}}^{\mu}$ couples to the source term $\omega_{\mu}^{\hat{a} \hat{b}}$ in the Lagrangian as $\mathcal{L}_{\text {source }}=\omega_{\mu}^{\hat{a} \hat{b}^{\mu}}{ }_{\hat{a} \hat{b}}$.

Precisely speaking, what we define above is "total angular momentum" current, rather than "spin" current. Note that only the total contribution of the angular momentum current, coming from both the orbital and the spin angular momentum, is conserved. A difficulty in dealing with spin transport phenomena is in the definition of the spin current, because the intrinsic spin is not conserved solely but rather conserved as a whole angular momentum. Therefore, the spin current, by itself, cannot be introduced as a conserved Nöther current at least in the relativistic limit. Thus, in this sense, the spin current defined above is slightly different from the conventional definition of the spin current often used in the nonrelativistic condensed-matter system, which includes the contribution of only the intrinsic electron spin.

We will also point out that it is possible that the orbital contribution gives only a subleading contribution, in the nonrelativistic limit. This is because the orbital angular momentum includes the spatial momentum as $\vec{L}=\vec{x} \times \vec{p}$. Thus, by taking an appropriate limit, the spin current, defined as a conserved one, may provide a good description of the spin transport. We will discuss how we take the nonrelativistic limit a bit more in detail in the discussion later.

There is a number of attempts to define the spin current in the literature. The original idea of using the spin connection as a source to obtain a spin current is found in Refs. [16,17], especially in $2+1$ dimensions. In Ref. [16] the authors treated the space and time separately and broke the Lorentz invariance explicitly. Another attempt to define a spin current is performed by introducing an SU(2)-valued gauge field, coupled to a spin degrees of freedom, in addition to a $U(1)$ electromagnetic field [18-20]. This SU(2) symmetry can be seen as a remnant of the local Lorentz symmetry, which is decomposed as $\mathrm{SO}(1,3) \cong \mathrm{SU}(2) \times \mathrm{SU}(2)$ in $3+1$ dimensions. However, since these $S U(2)$ are not decoupled except for the massless case, it is difficult to define the spin current as a conserved current only with the $\mathrm{SU}(2)$ gauge field. Actually, this $\mathrm{SU}(2)$ symmetry is broken in the presence of the spin-orbit interaction.

\section{HOLOGRAPHY}

Given the spin current definition in terms of spin connection, in order to study the spin current by the gauge/gravity duality scheme, we will evaluate the fluctuation mode of the spin connection. Note that holography induces one extra coordinate, i.e., a radial direction. So in the gravity side, the local Lorentz index runs as $\hat{a}=\hat{t}, \hat{x}, \hat{y}, \hat{z}$ and $\hat{r}$. Similarly the vector index runs $\mu=t, x, y, z, r$.

Before studying a component of the spin connection corresponding to a spin current in a spatial direction, we analyze a temporal component of a spin current $J_{t}^{\hat{x} \hat{y}}$, as an example. This term couples to $\omega_{t}^{\hat{x} \hat{y}}$. When the background metric is diagonal, the static contribution is calculated as

$$
\delta \omega_{t}^{\hat{x} \hat{y}}=\frac{1}{2} e^{x \hat{x}} e^{y \hat{y}}\left(\partial_{y} \delta g_{t x}-\partial_{x} \delta g_{t y}\right) .
$$

Here we apply a gauge choice $e_{r}^{\hat{a} \neq \hat{r}}=g_{r \mu \neq r}=0$. From the indices, it is clear that this represents a rotation of a metric fluctuation in the $x y$-plane. In terms of the gauge/gravity duality, the non-normalizable mode of this component is regarded as a chemical potential for the $\hat{z}$-component of the total angular momentum, i.e., $\omega_{t(\mathrm{NN})}^{\hat{x} \hat{y}}=\frac{1}{2} \mu^{\hat{z}}$, where the index (NN) represents the non-normalizable mode [21]. This chemical potential is naively interpreted as the difference between those for up and down spins, $\mu^{\hat{z}}=\frac{1}{2}\left(\mu_{\uparrow}-\mu_{\downarrow}\right)$. The $\hat{z}$-component spin density $J_{t}^{\hat{z}}$ corresponds to the normalizable mode of $\omega_{t(\mathrm{~N})}^{\hat{x} \hat{y}}$ in the holographic viewpoint, where the index $(\mathrm{N})$ represents the normalizable mode.

Similarly, let us study a fluctuation of the spin connection along the $x$-spatial direction, $\omega_{x}^{\hat{x} \hat{y}}$. This corresponds to a spin current $J_{x}^{\hat{x} \hat{y}}=\frac{1}{2} J_{x}{ }^{\hat{z}}$; i.e., $\hat{z}$-oriented spin flows along the $x$ direction. Here we can see that we need to turn on some of the off-diagonal elements of the background metric, in particular $g_{t x}$ and $g_{t y}$, which correspond to nonvanishing off-diagonal contributions of vielbeins, $e_{t}{ }^{\hat{x}}$ and $e_{t} \hat{y}$. To see this, assuming that the fluctuation depends only on $r$ and $t$ directions, we obtain

$$
\delta \omega_{x}^{\hat{x} \hat{y}}=-\frac{1}{2} e^{t \hat{x}} e^{y \hat{y}} \partial_{t} \delta g_{x y}+\frac{1}{2} e^{x \hat{x}} e^{t \hat{y}} \partial_{t} \delta g_{x x} .
$$

From this expression one can see that the off-diagonal components of the metric, $e_{t}^{\hat{x}}$ and $e_{t}^{\hat{y}}$, or equivalently $g_{t x}$ 
and $g_{t y}$, are required in order to give the spin current $J_{x} \hat{z}$. A physical meaning of this condition is discussed later.

\section{EXAMPLE: “BOOSTED” BLACK BRANE}

So far we have considered a boundary theory in $3+1$ $(\hat{x}, \hat{y}, \hat{z}$ and $\hat{t})$ dimensions. However, even if the boundary theory is $2+1$ dimensional, none of our argument so far is modified since $2+1$-dimensional theories still admit a spin along the " $z$ "-direction; Here $z$-direction is simply the $(\hat{a}, \hat{b})=(\hat{x}, \hat{y})$ component, $J_{\mu} \hat{x} \hat{y}$. We will conduct a calculation of the spin current in a holographic setting, but for simplicity of the calculation in the bulk, we consider a bulk theory in $3+1$ dimensions, which corresponds to a boundary theory in $2+1$ dimensions.

We demonstrate a calculation of the transport coefficients for spin with the simplest holographic setup, i.e., pure gravity in $3+1$ dimensions,

$$
\begin{gathered}
S=S_{\text {bulk }}+S_{\text {boundary }}, \\
S_{\text {bulk }}=\int d^{4} x \sqrt{-g}(R[g]-2 \Lambda), \\
S_{\text {boundary }}=2 \int d^{3} x \sqrt{-\gamma} \Theta,
\end{gathered}
$$

where the cosmological constant is $\Lambda=-3$, and $\gamma_{\mu \nu}$ is the boundary metric, defined by the metric components along the boundary dimensions. $\Theta$ is a scalar defined with the extrinsic curvature $\Theta^{\mu \nu}=-\frac{1}{2}\left(\nabla^{\mu} n^{\nu}+\nabla^{\nu} n^{\mu}\right)$, as $\Theta=\gamma_{\mu \nu} \Theta^{\mu \nu} . n^{\mu}$ is outward unit vector pointing along the radial direction. This boundary action is to provide a welldefined Dirichlet variational principle. In addition, we have to also take into account another counterterm, called the cosmological counterterm, which depends on the intrinsic curvature of the boundary [22]. Although this counterterm is important for the regulation of the boundary stress tensor, it is known that the correct boundary stress tensor, involving the contribution from the cosmological counterterm, can be read off simply from the normalizable modes of the metric [23]. As explained later, we will study the spin current in terms of the stress tensor based on the relation between the spin connection and the metric, and furthermore we will read off the boundary stress tensor from the normalizable modes. Therefore, we just apply the argument for the stress tensor, instead of taking the variation with the spin connection without worrying about the cosmological counterterm.

We study metric fluctuations around a boosted Schwarzschild black brane solution in $\mathrm{AdS}_{4}$,

$$
\begin{aligned}
d s^{2}= & -U(r) d t^{2}+\frac{1}{U(r)} d r^{2}+r^{2} d y^{2} \\
& +\left(r^{2}-a^{2} U(r)\right) d x^{2}-2 a U(r) d t d x
\end{aligned}
$$

with $U(r)=\left(r^{3}-r_{0}^{3}\right) / r . \quad r=r_{0}$ is the horizon while $r=\infty$ is the boundary. $r_{0}$ is related to the temperature $T$ as $T=3 r_{0} / 4 \pi$ [24]. This metric was obtained by a coordinate transformation $t \rightarrow t+a x$ on the AdSSchwarzschild solution, and it suffices for our purpose since it includes the off-diagonal metric element $g_{t x}$. We can check that this satisfies the Einstein equation $R_{\mu \nu}-\frac{1}{2} g_{\mu \nu} R+\Lambda g_{\mu \nu}=0$ and is not singular for $|a|<1$, and we can consider $a>0$ without loss of generality.

Let us perform a fluctuation analysis around the background solution. Fluctuations we consider are $\delta g_{t y}$ and $\delta g_{x y}$, and we assume the following form for ac fluctuations,

$$
\begin{gathered}
\delta g_{t y}=\delta g_{y t}=\epsilon e^{-i \omega t} r^{2} f(r), \\
\delta g_{x y}=\delta g_{y x}=\epsilon e^{-i \omega t} r^{2} h(r) .
\end{gathered}
$$

Then, nontrivial components of the Einstein equation to linear order in these fluctuations, $\mathcal{O}(\epsilon)$, are found to be just the ty-component, the ry-component and the $x y$-component. The other components of the Einstein equation turn out to be trivially satisfied. Among the three equations, the ry-component provides a constraint,

$$
f^{\prime}(r)=\left(a+\frac{r^{3}}{a\left(r_{0}^{3}-r^{3}\right)}\right)^{-1} h^{\prime}(r)
$$

where ' is for the $r$-derivative. With this relation, the ty-component reduces to a simple equation solely for $h(r)$,

$h(r)+\frac{r^{3}-r_{0}^{3}}{\omega^{2} r^{3}} \frac{d}{d r}\left[\frac{\left(r^{3}-r_{0}^{3}\right) r^{4}}{\left(1-a^{2}\right) r^{3}+a^{2} r_{0}^{3}} \frac{d}{d r} h(r)\right]=0$.

Furthermore, the remaining $x y$-component of the Einstein equations also reduces to the same equation (22). So, we just need to solve the equation (22) for $h(r)$ and relate it to $f(r)$ via the constraint equation (21). This equation (22), in the limit $a=0$, coincides with the equation for the shear viscosity calculation $[25,26]$.

Equation (22) can be written by a new coordinate $x \equiv r_{0} / r$ as

$\frac{\omega^{2}}{r_{0}^{2}} h(x)=x^{2}\left(x^{3}-1\right) \frac{d}{d x}\left[\frac{1-x^{3}}{x^{2}\left(1-a^{2}+a^{2} x^{3}\right)} \frac{d h(x)}{d x}\right]$.

The new coordinate $x$ ranging $0 \leq x \leq 1$ can make the boundary analysis easier.

Near the horizon $x=1$, we can solve (23) as

$$
h \propto \exp \left(-\frac{i}{3} \frac{\omega}{r_{0}} \log (1-x)\right)
$$

which amounts to the ingoing boundary condition at the horizon. Note that the equation of motion (23) and the ingoing boundary condition (24) depend on $r_{0}$ only through 
the combination $\omega / r_{0}$. Since $T \propto r_{0}$, the temperature dependence is the same as the $1 / \omega$ dependence. This is because the background is a finite temperature system of an AdS space, namely a scale invariant system, and therefore any nontrivial dependence comes from only the dimensionless ratio, $\omega / r_{0}$ [27].

Near the boundary $x=0$, we have two independent solutions of (23),

$$
\begin{gathered}
h=h_{0}\left(1-\frac{1}{2} \gamma x^{2}-\frac{1}{8} \gamma^{2} x^{4}+\cdots\right), \\
h=h_{3}\left(x^{3}+\cdots\right),
\end{gathered}
$$

with $\gamma(\omega, T) \equiv\left(1-a^{2}\right) \omega^{2} / r_{0}^{2}$. Here $h_{0}$ and $h_{3}$ are integration constants. We can find that $h_{0}$ is the nonnormalizable mode, while $h_{3}$ is the normalizable mode. Consider the bulk action, Eq. (16), and expand that around $r \rightarrow \infty$ in the background equation (18), with the fluctuation $h(r)$ and $f(r)$. After using the constraint (21), we find, to the quadratic order in $h(r)$, the leading $r$ behavior of the Einstein action is

$$
\begin{aligned}
& \left.\sqrt{-g}[R[g]-2 \Lambda]\right|_{r \rightarrow \infty} \\
& =(\text { background })-\frac{\epsilon^{2} e^{-2 i \omega t} r^{4}}{2\left(1-a^{2}\right)} h^{\prime}(r)^{2},
\end{aligned}
$$

neglecting the boundary terms. From this expression, we confirm that $h \sim$ const is the non-normalizable mode [28], while $h \sim r^{-3}$ is the normalizable mode.

We can also specify the boundary condition for the other fluctuation $f(r)$. From (21), we obtain

$$
f(x)=\int_{1}^{x} \frac{a\left(s^{3}-1\right)}{a^{2} s^{3}-a^{2}+1} \frac{d h(s)}{d s} d s+c,
$$

where $c$ is an integration constant. Near the horizon $x=1$, $h(x)$ approximated as (24) can give an ingoing wave for $f(x)$ only if $c=0$. So we need to put $c=0$, and $f(x)$ is uniquely determined once $h(x)$ is given. The magnitude $f_{0}$ of the non-normalizable mode of $f(x)$ can be read by (28) with $c=0$, while the magnitude $h_{3}$ of the normalizable mode of $f(x)$ is proportional to that of $h(x)$ (which is $h_{3}$ ), through (28).

\section{SPIN CURRENT AND STRESS TENSOR}

Let us pose and understand the physical meaning of the modes we consider above. The spin connection can be written with the metric, or the vielbein as Eq. (7). This means that the spin current, which is dual to the spin connection, should be associated with the stress tensor, which is dual to the metric. Therefore, we have to evaluate the spin current by taking into account its relation to the stress tensor. In other words, the spin current can be determined by comparing the coefficients appearing in the following relation:

$$
J_{\hat{a} \hat{b}}^{\mu} \delta \omega_{\mu}^{\hat{a} \hat{b}}=T^{\rho \sigma} \delta \gamma_{\rho \sigma}=\delta \mathcal{L} .
$$

Here $\mathcal{L}$ is the Lagrangian of the quantum field theory in the boundary $2+1$ dimensions. Note that these metric and spin connections are defined on the boundary, and therefore all the indices run without the radial direction. We have omitted the volume factor $\sqrt{-\gamma}$ for simplicity.

To obtain an explicit relation between the spin current and the stress tensor, we first need to choose a local Lorentz frame. Any spin current is dependent on the choice of the frame. The boundary metric is

$g_{t t}=-1, \quad g_{t x}=g_{x y}=-a, \quad g_{x x}=1-a^{2}, \quad g_{y y}=1$.

These are given by subtracting the scale factor $r$ of the bulk metric in the limit $r \rightarrow \infty$. A natural choice of the local Lorentz frame for the background vielbein consistent with this metric is given by [29]

$e_{t}^{\hat{t}}=1, \quad e_{x}^{\hat{t}}=a, \quad e_{x}^{\hat{x}}=1, \quad e_{y}^{\hat{y}}=1$

We turned on the ac fluctuation of the metric given by Eqs. (19) and (20), and the most generic vielbein fluctuation consistent with (19) and (20) is a set $\left\{e_{t}^{\hat{y}}, e_{x}{ }^{\hat{y}}, e_{y}{ }^{\hat{t}}, e_{y}{ }^{\hat{x}}\right\}$, which satisfies the two relations

$$
\begin{gathered}
e_{t}^{\hat{y}}-e_{y}^{\hat{t}}=\epsilon e^{-i \omega t+i k_{x} x+i k_{y} y} f_{0}, \\
e_{x}^{\hat{y}}+e_{y}^{\hat{x}}-a e_{y}^{\hat{t}}=\epsilon e^{-i \omega t+i k_{x} x+i k_{y} y} h_{0}
\end{gathered}
$$

coming from the constraint $\gamma_{\mu \nu}=e_{\mu}{ }^{\hat{a}} e_{\nu}{ }^{\hat{b}} \eta_{\hat{a} \hat{b}}$. Here we used Fourier modes as $\sim e^{-i \omega t+i k_{x} x+i k_{y} y}$, and $\left(\omega, k_{x}, k_{y}\right)$ is the frequency/momentum for the fluctuations. The other components of the vielbein are consistently put to zero in our case.

With this at hand, all nontrivial components of the spin connection are 


$$
\begin{aligned}
& \delta \omega_{t}^{\hat{t} \hat{y}}=i \omega e_{y}{ }^{\hat{t}}, \\
& \delta \omega_{t}{ }^{\hat{x} \hat{y}}=-\frac{i}{2} k_{x}\left(e_{t}^{\hat{y}}-e_{y}{ }^{\hat{t}}\right)+\frac{i}{2} \omega\left(e_{y}{ }^{\hat{x}}-e_{x}^{\hat{y}}+a e_{y}{ }^{\hat{t}}\right), \\
& \delta \omega_{x}{ }^{\hat{\imath} \hat{y}}=-\frac{i}{2} k_{x}\left(e_{t}^{\hat{y}}+e_{y}{ }^{\hat{t}}\right)+\frac{i}{2} \omega\left(-e_{y}^{\hat{x}}-e_{x}^{\hat{y}}+a e_{y}{ }^{\hat{t}}\right), \\
& \delta \omega_{x}{ }^{\hat{x} \hat{y}}=-\frac{i}{2} k_{x}\left(2 e_{y} \hat{x}+a e_{t}^{\hat{y}}-a e_{y}{ }^{\hat{t}}\right) \\
& +\frac{i}{2} a \omega\left(-e_{y}^{\hat{x}}-e_{x}^{\hat{y}}+a e_{y}^{\hat{t}}\right), \\
& \delta \omega_{y}{ }^{\hat{t} \hat{x}}=-\frac{i}{2} k_{x}\left(e_{t}^{\hat{y}}-e_{y}{ }^{\hat{t}}\right)+\frac{i}{2} \omega\left(-e_{y}{ }^{\hat{x}}-e_{x}{ }^{\hat{y}}+a e_{y}{ }^{\hat{t}}\right), \\
& \delta \omega_{y}{ }^{\hat{t} \hat{y}}=-i k_{y} e_{t}{ }^{\hat{y}}, \\
& \delta \omega_{y}{ }^{\hat{x}} \hat{y}=-i k_{y}\left(a e_{t}^{\hat{y}}-e_{x}^{\hat{y}}\right) .
\end{aligned}
$$

Keeping the two relations (32) and (33) satisfied, we can make a gauge choice of the local Lorentz frame, $e_{y}{ }^{\hat{t}}=e_{y}{ }^{\hat{x}}=0$, and restrict ourselves to homogeneous fluctuation, $k_{x}=k_{y}=0$. In this local Lorentz frame, the above spin connections are simplified, and all the nonzero components are

$$
\begin{gathered}
\delta \omega_{x}^{\hat{x} \hat{y}}=-\frac{i a \omega}{2} \epsilon e^{-i \omega t} h_{0}, \\
\delta \omega_{t}^{\hat{x} \hat{y}}=\delta \omega_{x}{ }^{\hat{t} \hat{y}}=\delta \omega_{y}{ }^{\hat{t} \hat{x}}=-\frac{i \omega}{2} \epsilon e^{-i \omega t} h_{0} .
\end{gathered}
$$

Since $h_{0}$ is the constant mode of the boundary metric $g_{x y}$, it is a source for the boundary stress tensor $T^{x y}$, and therefore we obtain the spin current coupled to the spin connection from this expression as

$$
\begin{gathered}
J_{x}^{\hat{x} \hat{y}}=-\frac{1}{a} \frac{1}{2 i \omega} T^{x y}, \\
J_{t}^{\hat{x} \hat{y}}=J_{x}{ }^{\hat{t} \hat{y}}=J_{y}^{\hat{t} \hat{x}}=-\frac{1}{2 i \omega} T^{x y} .
\end{gathered}
$$

All the other components, other than each antisymmetric partner $J_{\mu} \hat{b} \hat{a}=-J_{\mu} \hat{a} \hat{b}$, are zero. These combined with (35) and (36) clearly satisfy (29). $J_{x}^{\hat{x} \hat{y}}$ is the spin current along the $x$ direction, and $J_{t}{ }_{x}^{\hat{x}}\left(=J_{x}^{\hat{t} \hat{y}}=J_{y}{ }^{\hat{t} \hat{x}}\right)$ is the temporal component of the spin current, corresponding to the spin density.

Here we have employed a choice of the local Lorentz frame $e_{y}{ }^{\hat{t}}=e_{y}{ }^{\hat{x}}=0$. However, other local Lorentz frame choices are also possible. Actually, for a certain other choice of the local Lorentz frame, one can show that the spin current determined in this way is equivalent to a popular definition of the angular momentum current $M$ made by the stress-energy tensor,

$$
M_{\nu \lambda}^{\mu} \equiv x_{\nu} T_{\lambda}^{\mu}-x_{\lambda} T_{\nu}^{\mu} .
$$

Due to this relation, for example, we can obtain the normalizable and non-normalizable modes for the spin connection from those for the metric. Note that this current is with the target spacetime indices, so in order for this to be equivalent to our spin current $J$, a certain local Lorentz frame should be appropriately chosen.

To check this explicitly, we consider our case of nonzero $T^{t y}$ and $T^{x y}$. We consider $a=0$ for simplicity. From the definition (39), one obtains

$$
\begin{aligned}
& M_{t y}^{t}=-t T^{t y}, \quad M_{x y}^{t}=x T^{t y}, \quad M_{t y}^{x}=-t T^{x y}, \\
& M^{x}{ }_{x y}=x T^{x y}, \quad M^{y}{ }_{t x}=x T^{t y}-t T^{x y}, \\
& M^{y}{ }_{t y}=y T^{t y}, \quad M^{y}{ }_{x y}=-y T^{x y} .
\end{aligned}
$$

One can show that all of these are consistent with the spin connections (34) only when we choose a local Lorentz frame at which

$$
e_{t}^{\hat{y}}=-e_{y}^{\hat{t}}, \quad e_{x}^{\hat{y}}=e_{y}^{\hat{x}}
$$

are satisfied. To see this, in this case, (32) and (33) become

$$
\begin{aligned}
& e_{t}^{\hat{y}}=-e_{y}^{\hat{t}}=\frac{1}{2} \epsilon e^{-i \omega t+i k_{x} x+i k_{y} y} f_{0}=\frac{1}{2} \delta g_{t y}, \\
& e_{x}^{\hat{y}}=e_{y}^{\hat{x}}=\frac{1}{2} \epsilon e^{-i \omega t+i k_{x} x+i k_{y} y} h_{0}=\frac{1}{2} \delta g_{x y},
\end{aligned}
$$

and (34) becomes

$$
\begin{aligned}
& \delta \omega_{t}^{\hat{t} \hat{y}}=\frac{1}{2} \partial_{t} \delta g_{t y}, \quad \delta \omega_{t}^{\hat{x} \hat{y}}=-\frac{1}{2} \partial_{x} \delta g_{t y}, \\
& \delta \omega_{x}^{\hat{\hat{y}} \hat{y}}=\frac{1}{2} \partial_{t} \delta g_{x y}, \quad \delta \omega_{x}^{\hat{x} \hat{y}}=-\frac{1}{2} \partial_{x} \delta g_{x y}, \\
& \delta \omega_{y}^{\hat{t} \hat{x}}=-\frac{1}{2} \partial_{x} \delta g_{t y}+\frac{1}{2} \partial_{t} \delta g_{x y}, \\
& \delta \omega_{y}{ }^{\hat{\hat{y}}}=-\frac{1}{2} \partial_{y} \delta g_{t y}, \quad \delta \omega_{y}^{\hat{x} \hat{y}}=\frac{1}{2} \partial_{y} \delta g_{x y} .
\end{aligned}
$$

Therefore, the angular momentum current $M^{\mu}{ }_{\nu \lambda}$ given by (40) satisfies our previous anticipation (29) with the spin connection (44) via a partial integration.

The freedom for the local Lorentz frame choice corresponds to the freedom for the local choice of the axes to define the rotation for the angular momentum. Note that in any choice of the local Lorentz frame for the vielbein fluctuations, interestingly, the expression of the most important spin connection (35) is universal, and therefore so is (37).

\section{TRANSPORT COEFFICIENTS}

$h_{3}$ is proportional to the spin current $J_{x}^{\hat{z}}=2 J_{x}^{\hat{x} \hat{y}}$. $h_{0}$ is proportional to the spin gradient along the $x$ direction $\nabla_{x} \mu^{\hat{z}}$, 
because $\nabla_{x} \mu^{\hat{z}}=2 \nabla_{x} \omega_{t(\mathrm{NN})}^{\hat{x} \hat{y}}$ is gauge equivalent to $-2 \nabla_{t} \omega_{x(\mathrm{NN})}^{\hat{x} \hat{y}}=2 i \omega \omega_{x(\mathrm{NN})}^{\hat{x} \hat{y}}$.

$f_{0}$ corresponds to the thermal gradient along the $y$ direction due to the relation $i \omega \delta g_{t y}^{(\mathrm{NN})}=r^{2} \nabla_{y} T / T$ [30]. $f_{3}$ corresponds to a thermal current along the $y$ direction, since $\delta g_{t y}^{(\mathrm{N})}$ is dual to the stress tensor, $\delta g_{t y}^{(\mathrm{N})}=T_{t y}$.

From these, we can evaluate the spin transport coefficient $\alpha$ and the thermal spin Hall conductivity $\kappa_{s \mathrm{H}}$, defined as

$$
J_{x}^{\hat{z}}=-\alpha \nabla_{x} \mu^{\hat{z}}, \quad J_{x}^{\hat{z}}=-\kappa_{s \mathrm{H}} \nabla_{y} T \text {. }
$$

Using holography, these coefficients are represented by normalizable and non-normalizable modes as

$$
\begin{gathered}
\alpha=-\frac{J_{x}^{\hat{x} \hat{y}}}{i \omega \delta \omega_{x(\mathrm{NN})}^{\hat{x} \hat{y}}}=\frac{h_{3}}{i a^{2} \omega^{3} h_{0}}, \\
\kappa_{s \mathrm{H}}=-\frac{2 J_{x}^{\hat{x} \hat{y}}}{i \omega T \delta g_{t y}^{(\mathrm{NN})} / r^{2}}=-\frac{h_{3}}{a \omega^{2} T f_{0}} .
\end{gathered}
$$

As we have seen, the ratio $h_{3} / h_{0}$ and $h_{3} / f_{0}$ are functions of only $\omega / T$, in Eqs. (23), (24) and (28). We obtain these by solving the bulk equation and imposing the ingoing boundary condition at the horizon, and the radial $r$ dependence of the bulk equation is reflected as $\omega / r_{0}$ dependence in the boundary viewpoint.

Actually the sources $\left(h_{0}, f_{0}\right)$ and the expectation values $\left(h_{3}, f_{3}\right)$ are related by a 2 by 2 matrix, and the coefficients $\alpha$ and $\kappa_{\mathrm{sH}}$ are just the upper two elements of this 2 by 2 matrix. However, as we have seen, in our system it follows that $f_{3}=(a-1 / a)^{-1} h_{3}$ due to the relation (21), where $f_{3}$ is the normalizable mode coefficient for $f(r)$, just as $h_{3}$ in the equation (26). Therefore, the ratio $f_{3} / f_{0}$ and the ratio $f_{3} / h_{0}$ are essentially the same as $h_{3} / f_{0}$ and the ratio $h_{3} / h_{0}$.

We have evaluated these transport coefficients by a numerical method for solving the differential equation (23). By varying the frequency $\omega$, we find the ac conductivities as shown in Fig. 2 [31].

For the numerical simulations, we have worked in the unit $T=1$ and chosen $a=0.03, a=0.5$ and $a=0.9$ for simplicity. The top figure of Fig. 2 is the spin transport coefficient $\alpha$. This is the coefficient on the spin current $J_{x} \hat{z}$ as a response to the ac external gradient of the spin chemical potential $\mu^{\hat{z}}$. The bottom figure of Fig. 2 is the thermal spin Hall conductivity $\kappa_{\mathrm{sH}}$. In both figures, the transport coefficients are multiplied by $a^{2} \omega^{2}$ to show the $\omega / T$ dependence clearly. From the figures, we find that the imaginary parts $\times \omega^{2}$ vanish linearly at $\omega=0$, so around the origin the imaginary parts behave as $1 / \omega$. This means that in the real parts there exists a Drude peak proportional to $\delta(\omega)$ often observed in superconducting/ metal phases. We also see specific behavior of the thermal spin Hall conductivity, changing the sign of the transport
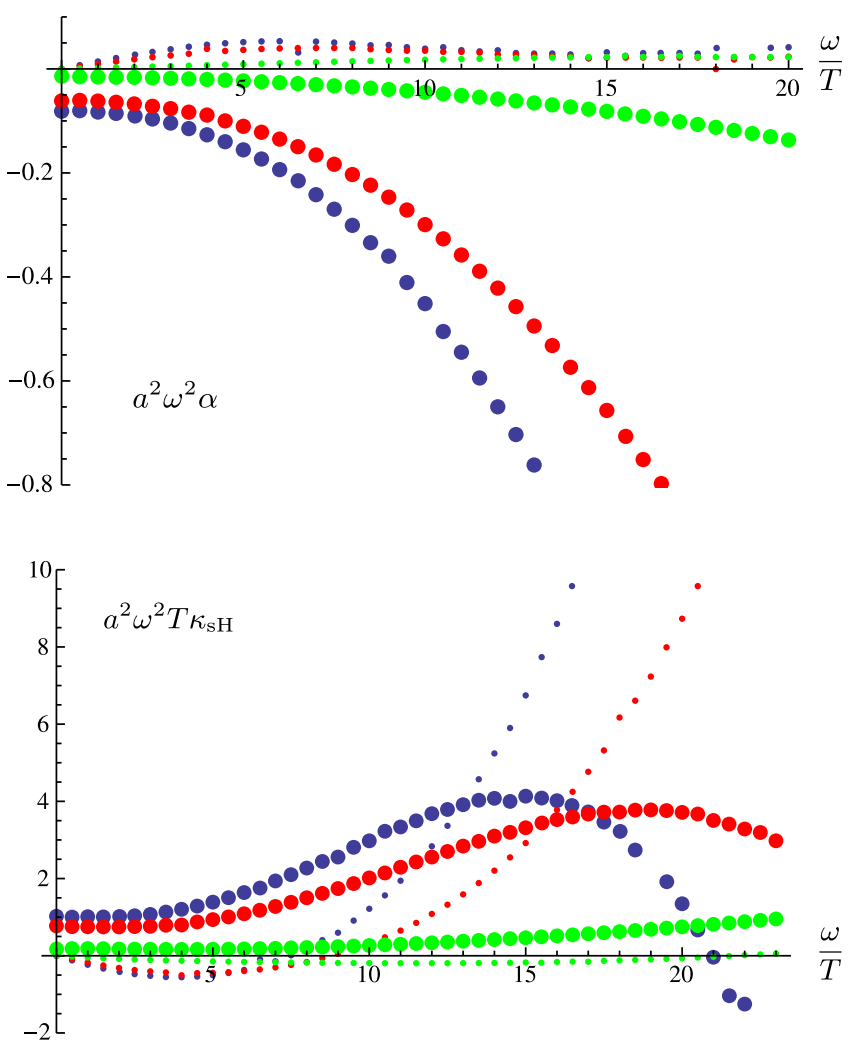

FIG. 2 (color online). Top: the spin transport coefficient $\alpha$ as a function of the frequency over the temperature, $\omega / T$. Large dots are the real part $\operatorname{Re}[\alpha]$, and small dots are the imaginary part $\operatorname{Im}[\alpha]$. Blue, red and green correspond to $a=0.03,0.5$ and 0.9 , respectively. Bottom: the thermal spin Hall conductivity $\kappa_{s \mathrm{H}}$ as a function of $\omega / T$.

coefficient as the frequency gets larger. It is quite interesting to observe such frequency dependence by experimental or other theoretical setups.

\section{ON THE SPIN CURRENT DEFINITION}

We have evaluated the spin current following the relation (29). However, (29) is not necessarily the same as our definition of the spin current (6). We will now discuss that the spin current evaluated by the definition (6) yields zero value, using the action (15) [32]. This is the reason why we need to relate the spin current to the stress tensor as (29), which we have used in this paper.

To obtain the spin current following the definition (6) in holography, note that (6) means that we have to differentiate the action (15) with the boundary spin connection, which is defined by the spin connections along the boundary directions. The contribution coming from a variation of the bulk action (16) by the boundary spin connection, vanishes by using the bulk equations of motion. Thus, the contribution to the spin current comes from a variation of the boundary action (17) only. However, we will see that this contribution also vanishes. 
Whenever we take a variation, we have to fix all the other quantities. In this case, we regard each of the boundary spin connection components as an independent degree of freedom, and then we take a variation of the action by that, while keeping all the other quantities, which include the metric, fixed. In this formulation, each spin connection component is an independent degree of freedom from the metric; the independent degrees of freedom are metric and spin connection. In fact, we can formulate general relativity in such a way, by i.e., the so-called Palatini formulation of gravity. However, this procedure turns out to give a vanishing spin current.

To see this, let us conduct a variation of the boundary action (17) by the boundary spin connection. The extrinsic curvature $\Theta$ is written with the normal vector $n_{\mu}$ as $\Theta=-\gamma^{\mu \nu} \nabla_{\mu} n_{\nu}$. In the Palatini formalism, the boundary metric $\gamma^{\mu \nu}$ and the boundary spin connection are independent, and therefore the contribution form the boundary action variation yields

$$
J^{\mu} \hat{a} \hat{b}=-2 \gamma^{\rho \sigma} \frac{\delta \Gamma_{\xi \lambda}^{\eta}}{\delta \omega_{\mu}^{\hat{a} \hat{b}}} \frac{\delta\left(\nabla_{\rho} n_{\sigma}\right)}{\delta \Gamma_{\xi \lambda}^{\eta}}=-2 e^{\mu}{ }_{\hat{a}} e^{\nu}{ }_{\hat{b}} n_{\nu} .
$$

Since $n_{\nu} \neq 0$ only when $\nu=r$ and $e_{\hat{b}}^{r} \neq 0$ only when $\hat{b}=\hat{r}$, there is no spin current on the boundary. This shows that the spin current evaluated by the Palatini formalism vanishes [33]. To obtain a nonvanishing spin current, we should not regard the metric and the spin connection as independent degrees of freedom. We need to modify our definition of the spin current (6) slightly.

Therefore, in this paper we do not regard the spin connection as an independent variable but associate it with the metric. This further implies that our spin current, which is dual to the spin connection, should be associated with the stress tensor, which is dual to the metric. In the Palatini formalism, the relation (7) comes from the equation of motion for the spin connection. Therefore, we have evaluated the spin current by taking into account its relation to the stress tensor as (29) in this paper.

\section{DISCUSSIONS : SPIN VS ANGULAR MOMENTUM}

In this paper we have investigated the spin transport phenomena from the viewpoint of gauge/gravity correspondence. We have introduced the proper definition of the spin current, as a conserved Nöther's current, which couples naturally to the spin connection.

We have analyzed the AdS Schwarzschild black brane geometry as a simple example to demonstrate how to study the spin transport in the context of the holography. We have calculated the spin transport coefficient $\alpha$ and the thermal spin Hall conductivity $\kappa_{\mathrm{SH}}$ by studying the fluctuations of the metric components. We have obtained the corresponding transport coefficient from the non-normalizable and normalizable modes propagating in the bulk gravity.

Let us comment on a physical meaning of the holographic analysis done in this paper. We have seen that the off-diagonal metric component for the background, i.e., $g_{t x}\left(=g_{x y}\right)$, is required for giving the spin current. Note that if there is such a component in the background geometry that leads to a constant energy flow coupled to $g_{t x}$. By applying the fluctuation $\delta g_{t y}$ in addition to the background flow, we should have an angular momentum current in the $x$-direction as shown in Fig. 3. It seems that our spin current almost corresponds to the orbital part of the angular momentum.

However, at least from the relativistic theoretical viewpoint, we cannot split the total angular momentum into contributions from orbital and intrinsic spin; spin is originally defined in the nonrelativistic system, where the Lorentz invariance is broken and we should treat space and time separately. Since in this paper we have considered the total angular momentum current defined in relativistic field theory, in order to really discuss the spin current, we need to take an appropriate nonrelativistic limit of our system. Only after taking that, we can extrapolate the spin contribution from the total angular momentum current, and we can discuss if the orbital contribution gives only a subleading contribution or not.

The nonrelativistic limit of relativistic conformal field theories is obtained by taking the discreet light-cone quantization (DLCQ). This limit reduces the boundary metric from AdS into the form [34-38]

$$
d s^{2}=-r^{2 z}\left(d x^{+}\right)^{2}+\frac{d r^{2}}{r^{2}}+2 r^{2} d x^{+} d x^{-}+r^{2} d \vec{x}^{2},
$$

where $x^{+}$is the light front time, and $r$ is the holographic radial direction as before. $x^{-}$is a new direction associated with the boost direction and we compactly $x^{-} \sim x^{-}+R$, and has an interpretation as "dual" to the conserved particle number since $P_{-}$is quantized as $N / R$, where $N$ is the particle number. $z$ is called the "dynamical exponent" and represents the difference of the scaling between time $x^{+}$and spatial coordinate $\vec{x}$.

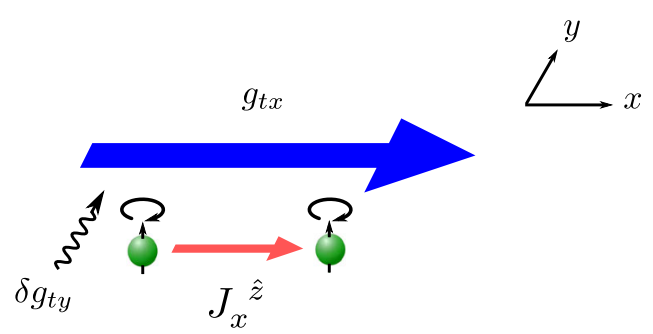

FIG. 3 (color online). When the off-diagonal background metric $g_{t x}$, namely a constant energy flow in the $x$-direction, is turned on, the angular momentum current as a spin current $J_{x} \hat{z}$ is induced by applying the fluctuation $\delta g_{t y}$. 
For example, starting from a boundary theory which is $3+1$ dimensional, we can obtain a $2+1$-dimensional nonrelativistic theory where we can identify $x^{+}=t+x^{3}$ and $x^{-}=t-x^{3}$. This metric possesses the Schrödinger symmetry for the $z=2$ case.

Taking this DLCQ limit, or simply replacing the boundary metric from $\mathrm{AdS}$ into the above, is not enough for extracting the spin information, since spin is not a conserved quantity by itself even here, and only the total angular momentum is a conserved one. To eliminate the contribution of the orbital angular momentum, it is best to consider a setting where the momentum of the particle is suppressed, namely an insulator. The insulator is realized as a system which has an energy gap. The energy gap is reflected in a holographic setting in the bulk as a system which has an IR cutoff, like the confinement in holographic QCD. The hard wall model is the simplest setting to realize the mass gap, and therefore this would lead one to a system which has an asymptotic metric as (49) and has an IR cutoff. Such a bulk setup is good for us to study the spin-transport phenomena, and it is interesting to see how the orbital and the real spin parts contribute to our total spin current, after taking the nonrelativistic limit.

In this paper we considered only the spin-current induction by the spin-current potential and also thermopotential, but not the one induced by an electric field. In real experiments, the spin current induced by some external electric field is more often considered, so this forces us to consider a bulk action coupled to the electromagnetic field. Adding impurity effects $[7,13,39,40]$ is also important. We hope to return to these analyses in the near future.

\section{ACKNOWLEDGMENTS}

N. I. would like to thank RIKEN Mathematical Physics Laboratory for kind hospitality where this project started. The research of K.H. is supported in part by JSPS Grants-in-Aid for Scientific Research No. 23105716, No. 23654096, and No. 22340069. The research of T. K. is supported in part by Grant-in-Aid for JSPS Fellows (Grant No. 23-593).
[1] S. Maekawa (ed.), Concepts in Spin Electronics (Oxford University Press, New York, 2006).

[2] I. Žutić and H. Dery, Nat. Mater. 10, 647 (2011).

[3] J. M. Maldacena, Adv. Theor. Math. Phys. 2, 231 (1998).

[4] S. Gubser, I. R. Klebanov, and A. M. Polyakov, Phys. Lett. B 428, 105 (1998).

[5] E. Witten, Adv. Theor. Math. Phys. 2, 253 (1998).

[6] F. Benini, C. P. Herzog, R. Rahman, and A. Yarom, J. High Energy Phys. 11 (2010) 137.

[7] S. Harrison, S. Kachru, and G. Torroba, Classical Quantum Gravity 29, 194005 (2012).

[8] F. Bigazzi, A. L. Cotrone, D. Musso, N. P. Fokeeva, and D. Seminara, J. High Energy Phys. 02 (2012) 078.

[9] C. P. Herzog and J. Ren, J. High Energy Phys. 06 (2012) 078.

[10] P. Benincasa and A. V. Ramallo, J. High Energy Phys. 06 (2012) 133.

[11] V. Alexandrov and P. Coleman, Phys. Rev. B 86, 125145 (2012).

[12] M. Luo, arXiv:1205.3267.

[13] K. Hashimoto and N. Iizuka, arXiv:1207.4643.

[14] T. Ishii and S.-J. Sin, J. High Energy Phys. 04 (2013) 128.

[15] For the spin to be approximately conserved, its coherence time must be sufficiently larger than its characteristic time scale.

[16] X. G. Wen and A. Zee, Phys. Rev. Lett. 69, 953 (1992).

[17] J. Fröhlich and U. M. Studer, Rev. Mod. Phys. 65, 733 (1993).

[18] V. Mineev and G. Volovik, J. Low Temp. Phys. 89, 823 (1992).

[19] A. S. Goldhaber, Phys. Rev. Lett. 62, 482 (1989).
[20] J. Fröhlich and U. Studer, Commun. Math. Phys. 148, 553 (1992).

[21] The factor $1 / 2$ is for a convenience due to the definition, Eq. (8).

[22] V. Balasubramanian and P. Kraus, Commun. Math. Phys. 208, 413 (1999).

[23] S. de Haro, S. N. Solodukhin, and K. Skenderis, Commun. Math. Phys. 217, 595 (2001).

[24] Our "boost" is simply a coordinate transformation. Since it is different from the Lorentz boost, it does not involve the $\gamma$ factor for a Lorentz transformation, and therefore the temperature does not change by this boost.

[25] P. K. Kovtun, D. T. Son, and A. O. Starinets, Phys. Rev. Lett. 94, 111601 (2005).

[26] Note that since these two equations solve all the Einstein equations these two modes, $\delta g_{t y}$ and $\delta g_{x y}$, decouple from the other components of the fluctuation. Therefore, this is a consistent truncation of the whole Einstein equations.

[27] The large $r / r_{0} \rightarrow \infty$ is equivalent to the $r_{0} \rightarrow 0$ with $r$ fixed, where the ratio $\omega / r_{0} \rightarrow \infty$ by fixing $\omega$. This implies that the bulk large (small) $r$ region corresponds to the large (small) $\omega$ in the boundary theory as in usual UV/IR correspondence [41].

[28] Note that terms like $r^{4} h^{\prime}(r)^{2}$ are equivalent to terms like $r^{2} h(r)^{2}$ through the integration by parts.

[29] When $a=0$, the vielbein is simply a unit matrix. The boost $t \rightarrow t+a x$ in the target space changes only the target space index $\mu$, resulting in this form of the vielbein.

[30] This can be derived by scaling time in the unit of temperature as $g_{t t}^{\text {boundary }}=-1 / T^{2}$ and by using a gauge 
transformation, which transform $\nabla_{x} g_{t t}^{\text {bulk }}$ to $-2 \nabla_{t} g_{t x}^{\text {bulk }}$. The extra $r^{2}$ is because of $g_{\mu \nu}^{\text {bulk }}=r^{2} g_{\mu \nu}^{\text {boundary }}$.

[31] One might wonder if Onsager's reciprocal relation holds in this case. Since we have two thermodynamical quantities represented by $f(r)$ and $h(r)$ in the holographic language, it is natural to argue the reciprocal relation. There are two points concerning the relation. First, since we have introduced the background ac external source for $g_{t y}$ and also the background metric $g_{t x}$, it is expected that we explicitly break the time-reversal symmetry. So there is no good reason for the reciprocal relation to hold in our case. Second, as we have noticed, once $h(r)$ is given, then $h(r)$ is completely determined. So, we cannot turn on the external source for $g_{x y}$ and $g_{t y}$ independently. This means that the Onsager reciprocal relation is not directly measured by our external sources.

[32] Here we do not take into account the cosmological counterterm for simplicity.
[33] In this evaluation, we have used the bulk equation of motion (7) to define $\Gamma_{\xi \lambda}^{\eta}\left(e_{\mu}^{\hat{a}}, \omega_{\mu}^{\hat{a} \hat{b}}\right)$. Instead, if we regard the extrinsic curvature $\Theta$ as being solely written by the vielbein, the variation (48) vanishes in the Palatini formalism.

[34] D. T. Son, Phys. Rev. D 78, 046003 (2008).

[35] K. Balasubramanian and J. McGreevy, Phys. Rev. Lett. 101, 061601 (2008).

[36] C. P. Herzog, M. Rangamani, and S. F. Ross, J. High Energy Phys. 11 (2008) 080.

[37] J. Maldacena, D. Martelli, and Y. Tachikawa, J. High Energy Phys. 10 (2008) 072.

[38] A. Adams, K. Balasubramanian, and J. McGreevy, J. High Energy Phys. 11 (2008) 059.

[39] S. A. Hartnoll and C. P. Herzog, Phys. Rev. D 77, 106009 (2008).

[40] A. Adams and S. Yaida, arXiv:1102.2892.

[41] A. W. Peet and J. Polchinski, Phys. Rev. D 59, 065011 (1999). 